\title{
Performance Improvement of Edge Detection based on Edge Likelihood Index
}

\author{
Xiaochen He and Nelson Hon Ching Yung \\ Department of Electrical and Electronic Engineering \\ The University of Hong Kong, Pokfulam Road, Hong Kong
}

\begin{abstract}
One of the problems of conventional edge detectors is the difficulty in distinguishing noise and true edges correctly using a simple measurement, such as gradient, local energy, or phase congruency. This paper proposes a performance improvement algorithm for edge detection based on a composite measurement called Edge Likelihood Index (ELI). In principle, given a raw edge map obtained from any edge detectors, edge contours can be extracted where gradient, continuity and smoothness of each contour are measured. The ELI of an edge contour is defined as directly proportional to its gradient and length, and inversely proportional to its smoothness, which offers a more flexible representation of true edges, such as those with low gradient, but continuous and smooth. The proposed method was tested on the South Florida data sets, using the Canny edge operator for edge detection, and evaluated using the Receiver Operator Characteristic curves. It can be shown that the proposed method reduces Bayes risk of ROC curves by over $10 \%$ in the aggregate test results.
\end{abstract}

Keywords: Edge detection, edge likelihood index, length, curvature, contour, receiver operating characteristics

\section{INTRODUCTION}

In the history of image processing, many edge detection methods have been devised, which differ in their purpose and in their properties. To summarize, it is observed that these methods typically compose of three sequential steps: differentiation, smoothing and labeling ${ }^{28}$. The first step of computing the derivatives of an image intensity function is often used to extract useful and representative edge information. However, it is well-known that derivatives are sensitive to noise and other local variations, which could produce a considerable amount of false edges. In a nutshell, it is not easy to design a method that will extract all the true or meaningful edges and not respond to other features that do not carry as much importance but have similar characteristics.

The second step of smoothing is designed to reduce the erratic effect of differentiation ${ }^{2}$, which in turns introduces a different set of problems, i.e., reduction in gradient peaks and displacement of prominent features in the image plane ${ }^{20}$. Therefore, the third step of labeling is required to localize edges and increase signal-to-noise ratio by suppressing false edges $^{28}$. In some of the early gradient detectors, edges were simply localized by thresholding the edge map. The classification of true or false edges is then based upon whether a selected property of the edge pixel is above or below yet another threshold. Although further improvement can be achieved by extracting local maxima of the gradient modulus along the direction of the gradient vector ${ }^{5}$, the selected property is usually a simple measure, such as gradient value or local energy. However, it is not necessary the case that true or meaningful edges must have, for example, higher gradient value than the noise or trivial features. As it is, some true edges may be regarded as false edges or trivial features including noise may be incorrectly recognized as true edges.

To address this issue, Canny used edge continuity as a criterion when performing the classification ${ }^{6}$. In essence, a low threshold is used in conjunction with a high threshold where pixels having gradient higher than the high threshold are considered as edge pixels. For those pixels with gradient below the high threshold, any of these pixels above the low threshold that can be connected to any pixels above the high threshold are then labeled as edge pixels. As a result, it generally gives a better set of detected edges, and is one of the popular edge detection methods around. The Canny approach proves that edge continuity does have a role in defining true edges. On the other hand, it is reasonable to assume that some weak but true edges may have gradient value higher than the low threshold but are not connected to any edge pixels above the high threshold, in which case, they will be discarded as non-edge pixels. This could well the case when all its connected pixels have gradients below the high threshold.

Visual Communications and Image Processing 2005, edited by Shipeng $\mathrm{Li}$,

Fernando Pereira, Heung-Yeung Shum, Andrew G. Tescher, Proc. of SPIE Vol. 5960

(SPIE, Bellingham, WA, 2005) $\cdot 0277-786 X / 05 / \$ 15 \cdot$ doi: 10.1117/12.633216

Proc. of SPIE Vol. 5960 59604W-1 


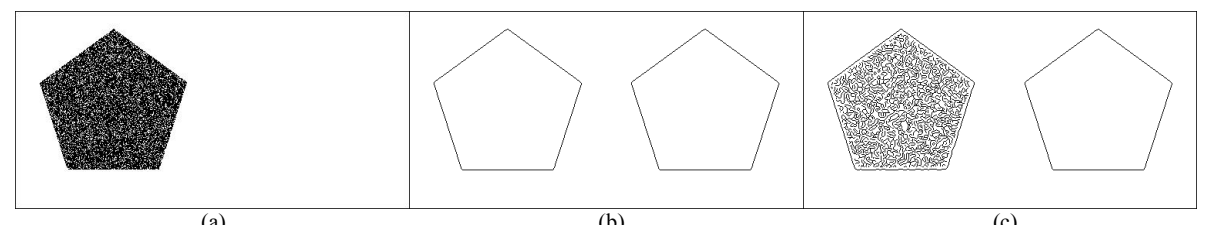

(a)

(b)

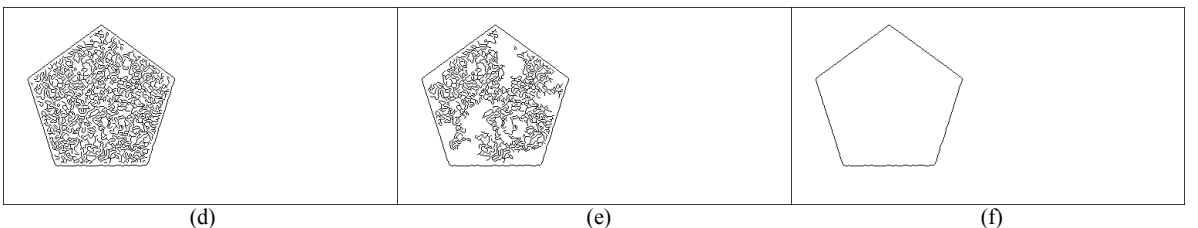

(d)

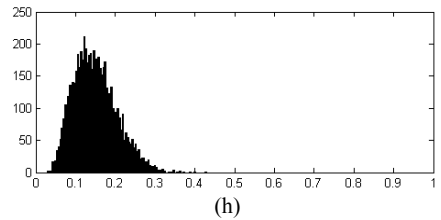

(g)

(h)

Fig.1: (a) Test image; (b) Ground truth of test image; (c) (f) Edge map of test image with different gradient thresholds: 0.1 for (c), 0.2 for (d), 0.3 for (e) and 0.5 for (f); (g) Gradient histogram of true edges; (h) Gradient histogram of false edges.

For example, as depicted in Fig.1(a), there are two pentagons in the test image with the right-hand one having lower gradient at the boundary and a smooth interior, while the left-hand one having higher gradient at the boundary and noisy spikes within. When we use a gradient based edge detector with relatively low threshold, both the pentagon boundaries are detected, but also abundant false edges appear inside the left-hand pentagon (Fig.1(c)). On the other hand, if we increase the threshold value gradually, the false edges within the right-hand pentagon can be eliminated eventually. However, the penalty is that the right-hand pentagon is not detected at all because of the high threshold (Fig.1(d)). Fig.1(c)-(f) depict the Canny edge maps for the test image with different thresholds: 0.1, 0.2, 0.3 and 0.5 respectively. Fig.1(g) and (h) depict the gradient histograms of true edges and false edges. From the histograms, we can see that the true edges of the right-hand pentagon fall within the same band as the noise and therefore cannot be separated properly.

The reason for this is that the conventional approach discussed so far concerns the choice of the simple measure. When a gradient operator is used, the measure would be the gradient modulus ${ }^{6,21,22,24,15}$; when the local energy model is used, the measure would be the phase congruency modulus ${ }^{17}, 18,13,14$. However, none of these measurements can solely represent the property of edges in totality. When a particular measure is chosen, some of the true edges may have values lower than say the trivial features, which will result in them being eliminated instead of the trivial features.

We believe that if other properties of these edges can be utilized, then the performance of edge detection will be enhanced. The question is thus what other properties should be considered and how should they be utilized? Our argument is that true edges are usually represented by significant and consistent local intensity variations. For instance, a meaningful edge normally has certain length and smoothly changing curvature, apart from its gradient magnitude and direction. Although this argument is not totally encompassing as there may be edges that are short with high curvature, such edges tend to associate with fine or textural features or noise in an image, which in fact do not have strong impact if they are not detected. Conversely, if they are detected, they would need to be removed or ignored at a later stage. Therefore, it will not be harmful if these edges are not extracted at the edge detection stage.

On this basis, we propose to describe an edge by a composite measure of the gradient, length (or continuity), and curvature (or smoothness). We call this description the Edge Likelihood Index (ELI). Given a raw edge map obtained from any traditional gradient-based edge detector with relatively low threshold (so that most of the true and false edges are retained), edge contours are extracted and for each contour its gradient, length and curvature are calculated, details of which will be explained in Section 2. The ELI of an edge contour is defined as directly proportional to the gradient and length of the contour and inversely proportional to its curvature. Furthermore, these variables are raised to the power of $p$, $q$ and $r$, respectively for optimization purpose. Finally, an empirical threshold of $E L I$ is used to set apart true edges from false edges. Compare this approach with those that use a simple measure, the consideration of edge length and curvature helps to identify edges more accurately, and there is more flexibility in the proposed method, as the parameters $p, q$ and $r$ in the $E L I$ definition can be tuned. Consequently, it can produce a more accurate edge map, with little trivial features and 
noise.

To investigate the performance of the proposed method, an objective evaluation method is needed. Previous and recent research literatures in this area are plentiful ${ }^{1,10,9,19,23,7,25}$. Bowyer et $\mathrm{al}^{4,26}$ empirically evaluated the performance of a set of eight edge detectors on the South Florida data set by using receiver operating characteristic (ROC) curves, which was a broadly used evaluation technique in computer vision. Our proposed method was also tested on the South Florida data sets, and evaluated using Bowyer's method. From the evaluation result, the proposed method can reduce Bayes risk of ROC curves by over $10 \%$ in aggregate test results.

This paper is organized as follows: in Section 2, our proposed method is presented in detail. Section 3 describes the evaluation method we have adopted. In Section 4, we depict the experiment result and provide an analytical discussion of the results, as well as the setting of the parameters. Conclusion of our investigation and proposal is given in Section 5.

\section{DETAILS OF THE METHODOLOGY}

As depicted in Fig.2, the proposed methodology relies on a raw gradient map and an edge map generated by any traditional edge detectors. The only requirement of this detector is that a low enough threshold is set so that most of the edges, true or false, are retained in the maps. Given the edge map, each contour is considered in turns, from which three parameters are computed, namely, contour mean gradient, length and curvature. The mean gradient represents the general intensity characteristic of the contour. As it is not the only parameter evaluated, true edges may have low mean gradient values, and yet still being classified as edge. The length of a contour is an important factor to determine whether it is a true edge or not. In fact, a long contour invariably indicates a true edge, while short contours are less indicative. Short contours mostly likely represent information that may not be meaningful. The curvature represents the flatness of the contour. Contours that have low mean curvature are more likely to represent a true edge. However, if a contour is round, it may be judged as non-edge due to its relatively high curvature. Therefore, the ELI is a measure that fuses the three parameters to give a more balanced evaluation of meaningful edges.

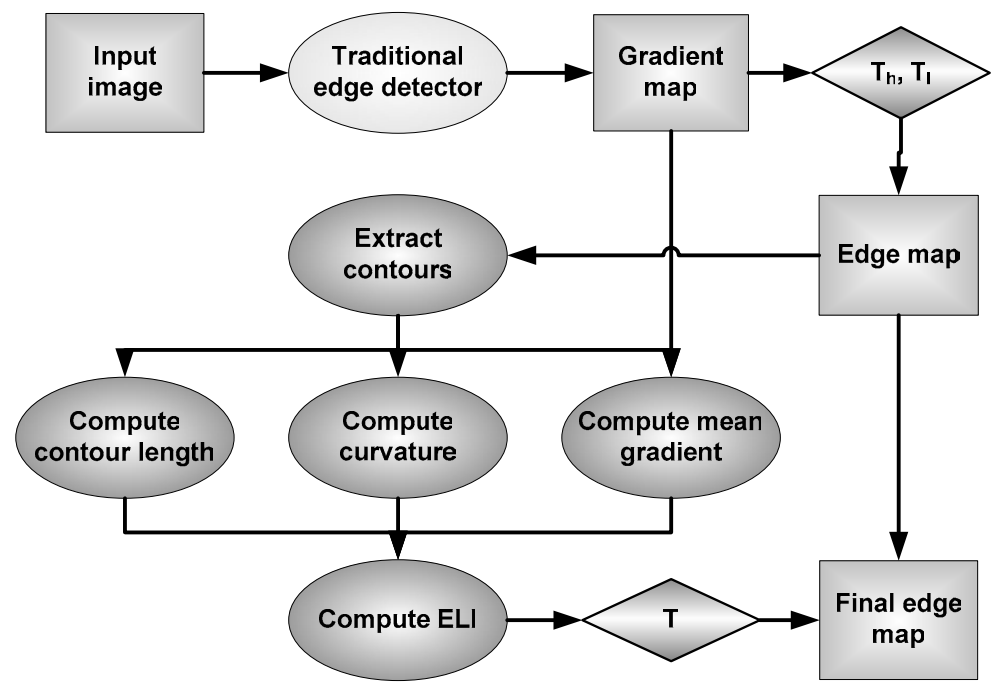

Fig. 2: Flow of the proposed method

In our actual experiment, Canny $^{6}$ edge detector was selected to produce the raw maps because of its good detection performance. The following lists the main algorithmic steps of our proposed algorithm:

1. Apply Canny edge detection (Eqt(1)) using relatively lower thresholds (sigma=1, high=0.05 and low=0) and generate the corresponding gradient map $(G M)$ and edge map $(E M)$.

$$
[G M, E M]=\text { Canny }(\text { low, high, sigma })
$$

2. Extract image edge contours from the edge map: First, all edge pixels which connected each other are grouped together into sequences by performing an 8 -connected component labeling operation ${ }^{8}$. Then these sequences are split 
at junction to get contours. The splitting operation is to make these contours not to be bifurcate. Finally, the endpoints of contours are checked to merge successive contours and we can get an edge contour set:

$$
\text { Contour_set }=\left\{C_{1}, C_{2}, \cdots, C_{m}\right\}
$$

where, $C_{i}=\left\{P_{1}^{i}, P_{2}^{i}, \cdots, P_{N_{i}}^{i}\right\}, P_{k}^{i}=\left(x_{k}^{i}, y_{k}^{i}\right), m$ is the total number of contours, $N_{i}$ is pixel number of the $i^{\text {th }}$ contour, and $\left(x_{k}^{i}, y_{k}^{i}\right)$ is the coordinate position of the $\mathrm{k}^{\text {th }}$ pixel in the $\mathrm{i}^{\text {th }}$ contour. It should be noted that all the extracted contour pixels should be an edge pixel in $E M$.

3. Evaluate the length of each contour, for a contour $C_{i}=\left\{\left(x_{1}^{i}, y_{1}^{i}\right),\left(x_{2}^{i}, y_{2}^{i}\right), \cdots,\left(x_{N_{i}}^{i}, y_{N_{i}}^{i}\right)\right\}$, define:

$$
\text { length }=N_{i}
$$

4. Compute mean gradient of contour:

$$
\text { gradient }_{i}=\frac{1}{N_{i}} \sum_{k=1}^{N_{i}} G M\left(x_{k}^{i}, y_{k}^{i}\right)
$$

5. Compute curvature of the contours based on the Curvature Scale Space (CSS) ${ }^{16}$ technique, where curvature $\kappa$ was defined as following:

$$
\mathcal{K}(u, \sigma)=\frac{\dot{X}(u, \sigma) \ddot{Y}(u, \sigma)-\ddot{X}(u, \sigma) \dot{Y}(u, \sigma)}{\left(\dot{X}(u, \sigma)^{2}+\dot{Y}(u, \sigma)^{2}\right)^{1.5}}
$$

and where $\dot{X}(u, \sigma)=x(u) \otimes \dot{g}(u, \sigma), \quad \ddot{X}(u, \sigma)=x(u) \otimes \ddot{g}(u, \sigma), \quad \dot{Y}(u, \sigma)=y(u) \otimes \dot{g}(u, \sigma), \quad \ddot{Y}(u, \sigma)=y(u) \otimes \ddot{g}(u, \sigma)$,

and $\otimes$ is the convolution operator, while $g(u, \sigma)$ denotes a Gaussian function with deviation $\sigma$. And then compute mean curvature of contour:

$$
\text { curvature }_{i}=\frac{1}{N_{i}} \sum_{u=1}^{N_{i}} \mathcal{K}(u)
$$

6. Compute the ELI using the following equation:

$$
E_{i}=\text { gradient }_{i}{ }^{p} \times \text { length }_{i}{ }^{q} / \text { curvature }_{i}^{r}
$$

where $p, q$, and $r$ are variables for optimization.

7. Normalize $E L I$ as follows:

$$
N E L I_{i}=E L I_{i} / \max (E L I)
$$

8. Using NELI to find meaningful edges, and deduce the final edge map (FEM):

$$
\text { For a pixel }(x, y) \in C_{i}, \operatorname{FEM}(x, y)= \begin{cases}1 & \text { if } N E L I_{i} \geq T \\ 0 & \text { if } N E L I_{i}<T\end{cases}
$$

It should be noted that the threshold $T$, and the exponents $p, q, r$ in Eqt(7) are adjustable parameters in the proposed method. It is found that when we set $p=q=r=1$, true edges with gradient lower than noise can be identified and detection performance for most of images can be improved. On the other hand, the detection result can be further tuned if these parameters are adjusted per image. The threshold $T$ can be determined empirically or determined automatically as in other traditional edge detectors.

\section{EVALUATION METHODOLOGY}

\subsection{Preliminary Evaluation}

Fig.3(a) depicts one of the test images from the South Florida data set, which the Canny edge map and the edge map for the case of $p=q=r=1$ and $T=0.08$ are shown in Fig.3(c) and (d), respectively, where the ground truth is shown in Fig.3(b). Generally, the proposed method detected most of the prominent edges with a few exceptions, one of which is the back edge of the brick. This edge is long with small curvature, but its gradient value is so low that its ELI is not large enough 


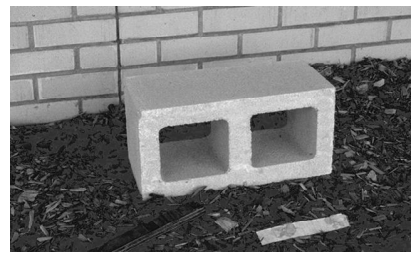

(a)

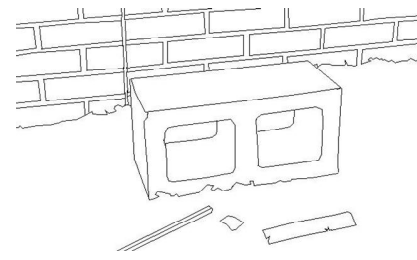

(b)

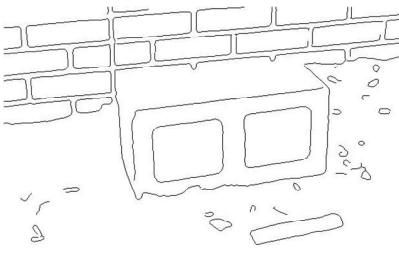

(c)

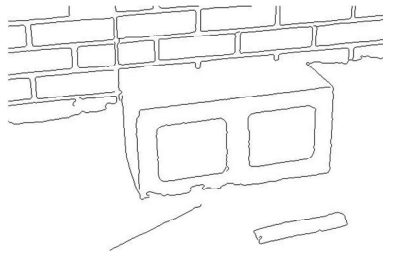

(d)

Fig.3: A test example of Canny and ELI (a) Test image; (b) Ground truth; (c) Canny detection result with $H=0.35, L=0.2, \sigma=2$; (d) ELI detection result with: $p=1, q=1, r=1, T=0.08$.

to be included as a meaningful edge. Another example is the small wood chip at the lower center of the image. Although its gradient intensity is reasonable, its short length and high curvature cause it to be discarded. On the other hand, the piece of black stick on its left was detected because of its long and straight feature. Compare this with the Canny edge map, it is found that Canny suffers from similar problem, for instance, it missed two edges at the top left-hand corner, the back edge of the brick, the back edges of the two holes and the long black stick at the bottom left. However, it is able to detect the small wood chip at the lower center. The major issue of the Canny detection is the detection of numerous fine features of the wood chips on the ground, populated on the lower half of the image. Although straightly speaking these can be classified as edges, their contribution to the interpretation of the image content however is questionable. Compare the two edge maps with the ground truth, it appears that Fig.3(d) resembles more closely to it than Fig.3(c). However, visual inspection is primarily subjective. We therefore need a more objective method for a detailed evaluation.

\subsection{Real and Ground Truth Images}

In terms of test images, we have chosen the South Florida data set because of their complexity. The images in the set are approximately 512 by 512 pixels, with 256 levels of grey levels. There are broadly divided into two categories. The first category contains "object images" taken from typical surroundings; and the second category contains "Aerial images" of both oblique and vertical views. The data set also contain the ground truth of these images, which were manually created for each image, in the form of specified edges that should be detected and specified regions in which no edges should be detected.
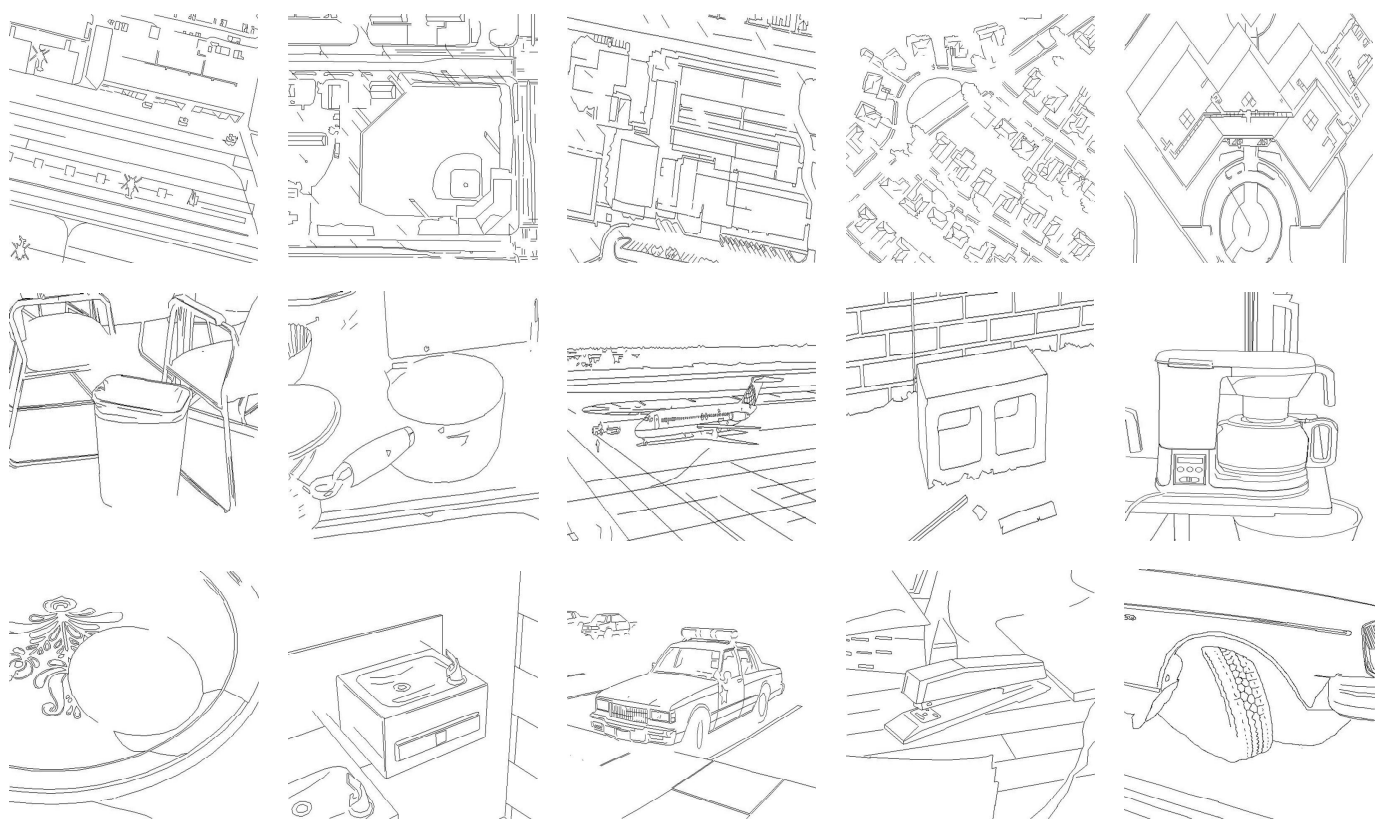

Fig.4: Ground truth of test images 
It should also be noted that the South Florida images have a 3-value ground truth. The three values are: (1) edge; (2) non-edge (background); and (3) pixels close to edges and some texture regions in the background. The pixels in the $3^{\text {rd }}$ set are not counted in the evaluation. These criteria can be criticized as giving a misleading measure of the performance of an edge detector ${ }^{11}$. In our evaluation, as shown in Fig.4, we reclassify the South Florida pixels as either edge or non-edge (which is the union of the $2^{\text {nd }}$ and $3^{\text {rd }}$ sets).

\section{EXPERIMENTAL RESULTS AND ANALYSIS}

\subsection{Comparison with the Canny detector}

In Bowyer's papers ${ }^{4,26}$, Canny was considered as one of the best edge detectors. Therefore, in this section, ROC curves of Canny detection and Canny detection with ELI (C-ELI) are presented and studied. As we only used the edge and non-edge values in the ground truth, the FN and FP value are larger than those presented in Bowyer's research.

In this evaluation, we attempted to set fine enough parameter sets for both methods in order to determine the best detection performance in the ROC curves. For Canny detection, Sigma was selected from 0.5 to 2.0 by step of 0.5 , high was from 0 to 1 by of step 0.05 , and low was from 0 to high by step 0.05 as well. For the $C$-ELI detector, a $4 \times 4 \times 4 \times 11$ parameter matrix was defined to describe the combinations of $p, q, r$ and $T$, where $p, q, r$ varied from 0.5 to 2.0 by step of 0.5 , and $T$ varied from 0 to 0.2 by step of 0.02 .

A set of 15 images was selected from the South Florida date set for evaluation, including object images and aerial images. Among these images, the block image is further selected to show the evaluation results, which (Fig.3(a)) contains long straight edges and some other trivial details. Fig.5(b) is the aggregated result of all the 15 test images produced by averaging individual ROC curves. In the aggregate test result, the proposed method reduces Bayes risk by $11 \%$ and reduces AUC by $4 \%$. For the block image, these two measures are reduced by $59 \%$ and $62 \%$ respectively. It appears that long straight edges as evident in the block image benefit more from the proposed method.

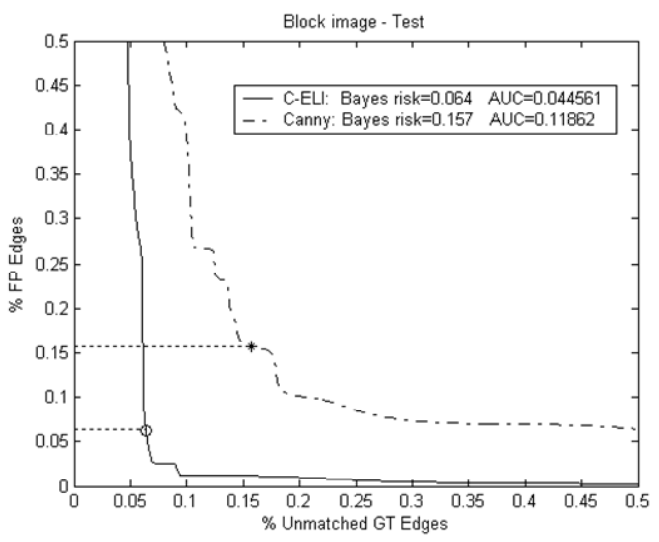

(a)

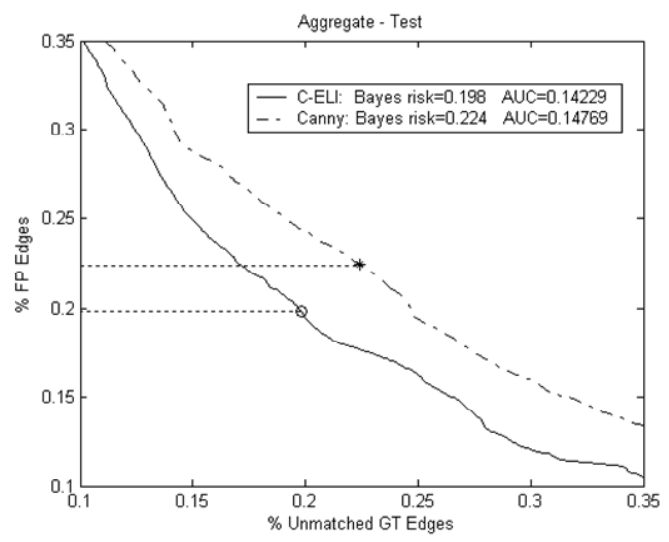

(b)

Fig.5: ROC curves (a) For the block image; (b) Aggregate result of a set of test images.

Fig.6-8 depict some of the detected edge maps. In these figures, the results with the smallest sum of FN and FP of $C$-ELI and Canny are shown in (a) and (b) respectively, and in general, the ELI results have better performance than the Canny results. In the block image, the back holes of the brick are weak and hard to detect. They do not appear in the Fig.6(b) since they have lower gradient than the boundary of the wood chips. On the other hand, these edges are detected by the $C$-ELI method (Fig.6(a)), because of the higher $E L I$ value due to their length and smoothness. The back edge at the top of the brick is another weak edge which has low gradient. Even the $C$-ELI method has difficulty detecting the complete edge line. This is because the edge is so weak that it was not even included in the raw edge map. Yet another example to demonstrate the power of the proposed method can be seen at the top left-hand corner of the image, where Canny missed some of the long edges, but the proposed method retained them all. Furthermore, false edges derived from the wood chips appear in the Canny result, but are suppressed by the proposed method. Similarly in Fig.7, boundary of the egg top was disjointed in the Canny result, while the $C$-ELI result detected all the meaningful edges. In Fig.8, the $C$-ELI result depicted a clear suppression of texture features in the bottom-left corner and detection of the complete 


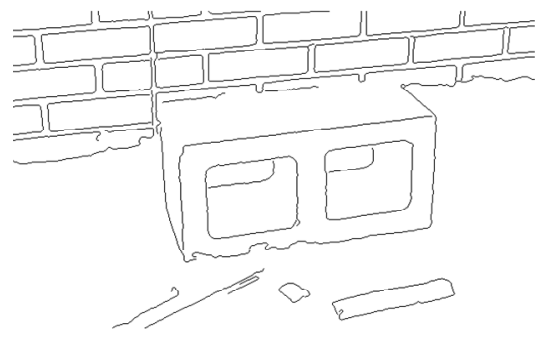

(a)

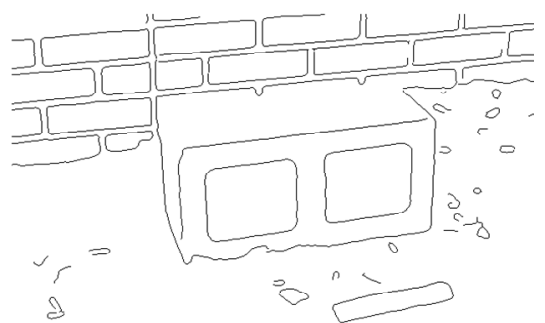

(b)

Fig.6: Best result of Block image (a) ELI detection result with smallest FN and FP: $p=0.5, q=1, r=2, T=0.04$, and FN=0.071, $\mathrm{FP}=0.026$; (b) Canny detection result with smallest $\mathrm{FN}$ and $\mathrm{FP}: H=0.35, L=0.2, \sigma=2$, and $\mathrm{FN}=0.183, \mathrm{FP}=0.109$

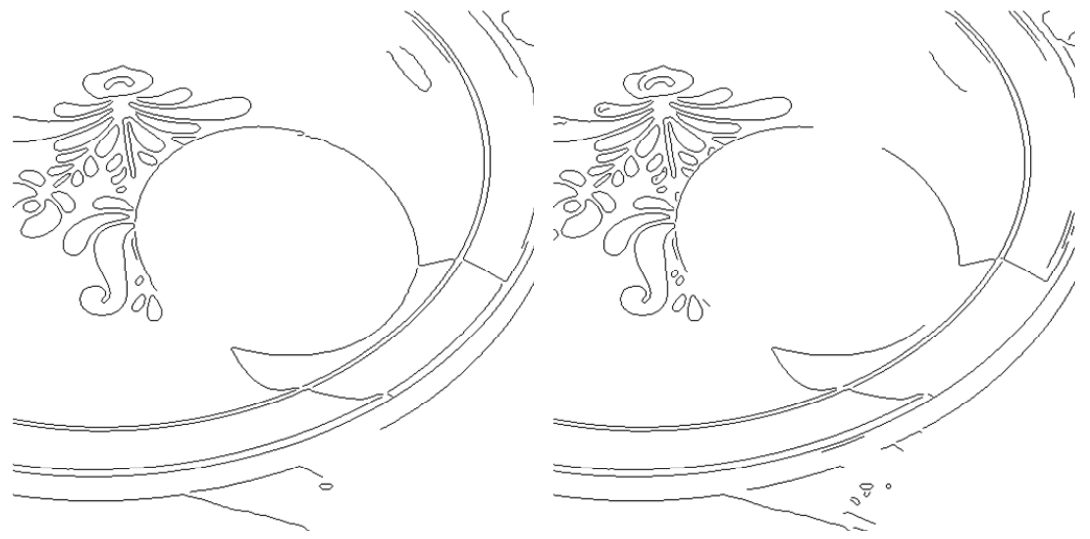

(a)

(b)

Fig.7: Best result of Egg image (a) ELI detection result with smallest FN and FP: $p=2, q=0.5, r=0.5, T=0.06$, and FN=0.074, $\mathrm{FP}=0.066$; (b) Canny detection result with smallest $\mathrm{FN}$ and $\mathrm{FP}: H=0.25, L=0.15, \sigma=1$, and $\mathrm{FN}=0.076, \mathrm{FP}=0.077$

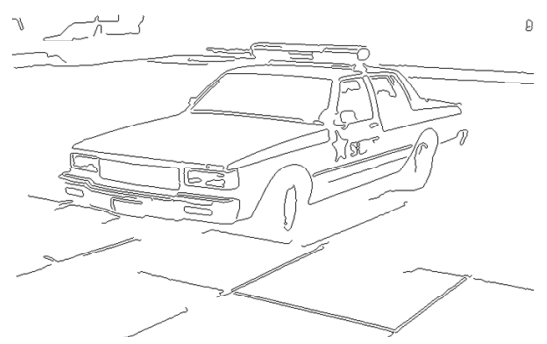

(a)

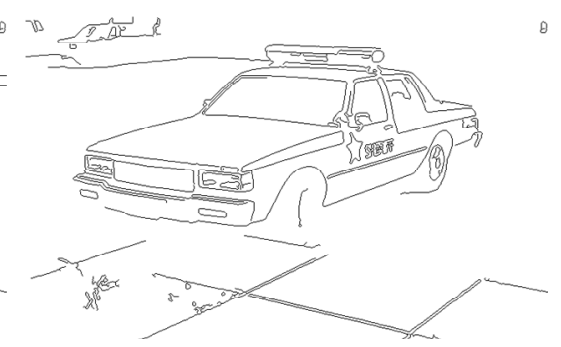

(b)

Fig.8: Best result of Vehicle image (a) ELI detection result with smallest FN and FP: $p=1.5, q=1, r=0.5, T=0.02$, and FN=0.259 $\mathrm{FP}=0.197$; (b) Canny detection result with smallest $\mathrm{FN}$ and $\mathrm{FP}: H=0.45, L=0.1, \sigma=1$, and $\mathrm{FN}=0.324, \mathrm{FP}=0.221$.

vehicle front-wheel. However, it should be noted that $C$-ELI performed poorly for some images, e.g. plane image. This is because the object in the image has many detailed features and the resolution of the image is relatively low. Meaningful edge features, noise and other trivial features intermix and cannot be distinguished appropriately by the proposed method.

\subsection{Initial Threshold}

As depicted in Fig.6(a), the back edge at the top of the brick was not detected. In fact, it was discovered that the edge was missing in the input edge map. This problem may be alleviated by reducing the initial threshold, which would result 


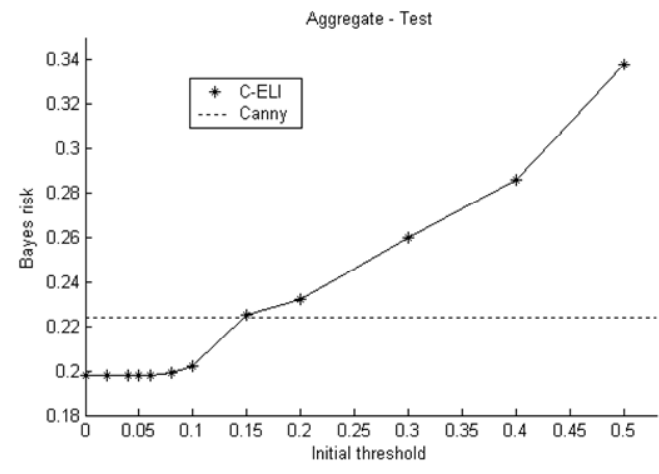

(a)

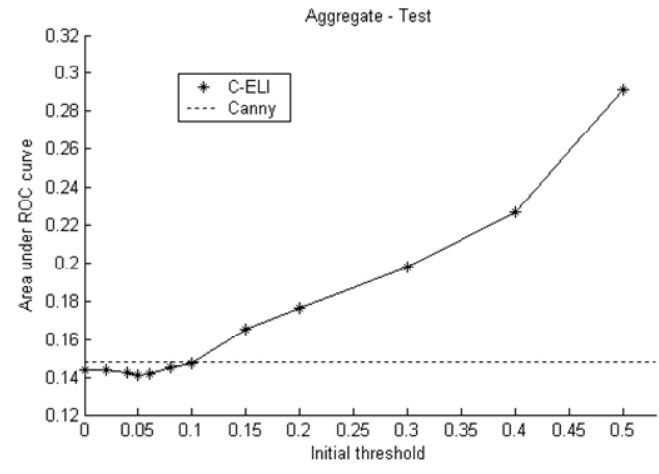

(b)

Fig.9: ROC parameters with different initial threshold. (a) Bayes risk; (b)AUC.

in more edges detected, but also an increase in computational complexity for contour extraction. To investigate the impact of the threshold on the $C$-ELI detection, we generated a number of aggregate ROC curves for different threshold values and computed their Bayes risk and AUC, and plotted them in Fig.9. In the experiment, the high threshold of Canny was set as different values from 0 to 0.5 , and the low threshold of Canny was always set to 0 . As shown in Fig. 9(a), the C-ELI detector consistently has lower Bayes risk than the Canny detector for threshold values lower than or equal to 0.1. Moreover, the Bayes risk is rather constant in this range. This implies that as long as the initial threshold is reasonably low, the $C-E L I$ detector is not too sensitive to changes in this value. In terms of AUC as depicted in Fig.9(b), a minimum is achieved when the initial threshold is around 0.05 . Below this, the AUC values are slightly higher, while above this, the AUC values are much higher than the Canny's. This can be explained as when the initial threshold is below 0.05 , there are many spurious edges in the input edge map that tend to connect with the meaningful edges, which then cause problem to the $C-E L I$ detector to separate them. On the other hand, if the initial threshold is high, meaningful edges may be removed from the input edge map, hence reducing the effectiveness of the C-ELI detector. Therefore, an initial threshold of 0.05 was adopted for the rest of our evaluation.

\subsection{Parameters of ELI}

In the proposed method, threshold $T$ and the exponents $p, q$ and $r$ are adjustable parameters, where $T$ can be determined empirically or automatically. In the Matlab version of Canny's edge detector, the default values of high threshold are suggested to be set just higher than the gradient strength of $70 \%$ of the image pixels, so that the detected edge pixels will have around $30 \%$ of overall pixels. Similarly, default threshold of ELI can be determined automatically as follows: Sort the pixels in raw edge map according to the ELI values, and then determine the threshold that gives true edges corresponding to $3.5 \%$ of the whole image. The value $3.5 \%$ is the mean edge pixel content in all the test images' ground

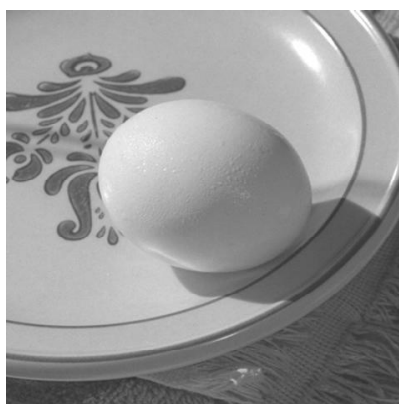

(a)

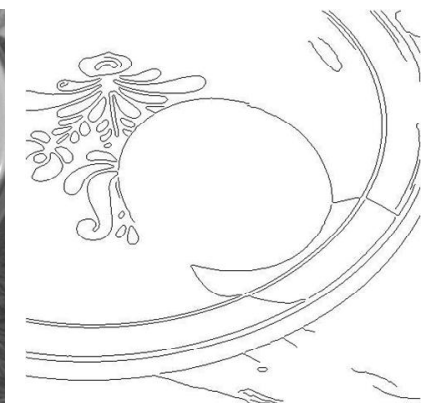

(b)

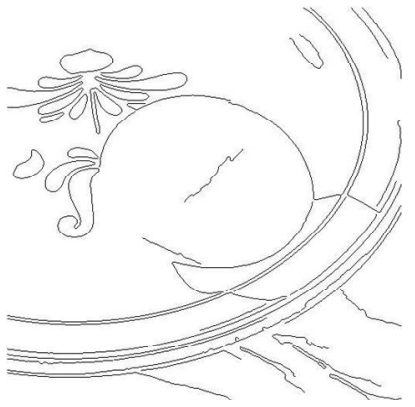

(c)

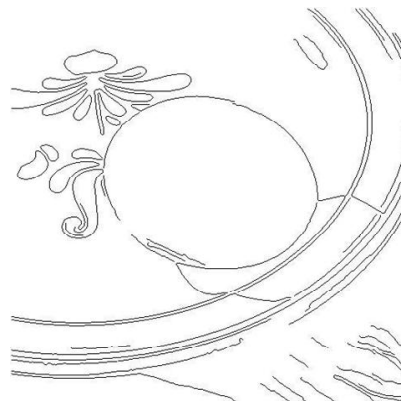

(d)

Fig.10: A test example with automatic threshold and different $p, q, r$ : (a) Original image; (b) ELI detection result with: $p=2, q=0.2$, $r=0.2, T=0.054577$; (c) ELI detection result with: $p=0.2, q=2, r=0.2, T=0.013879$; (d) ELI detection result with: $p=0.2, q=0.2, r=2$, $T=0.12746$. 
truth. This $3.5 \%$ is lower than Canny's 30\%, because the former is final edge map and the latter is followed by a non-maximum suppression operation.

For the exponents $p, q$ and $r$, if we simply set $p=q=r=1$, the proposed method can find true edges whose gradient is lower then noise, and enhance detection performance for most of the images tested. If we adjust these parameters for every image, the method has the potential of obtaining more accurate edge labeling result. Furthermore, different edge styles may be detected by appropriately adjusting the parameter values. Fig.10 presents a test example with automatic threshold and different $p, q, r$. Gradient, length, and curvature are emphasized in (b), (c) and (d) respectively. As a result, some fine patterns on the plate are detected in (b), but not in (c) and (d) due to their high gradient, but either short or having high curvature. However, when length is emphasized (Fig.10(c)), the transition from bright to dark on the egg's surface results into an edge because of its length rather than its gradient. Whereas the high curvature contours associated with the pattern printed on the plate are omitted when length is emphasized. The case of emphasizing curvature only highlights curvature as depicted in Fig. 10(d). The bottom-left boundary of the egg, which has very low gradient and is also broken into short segments, is neither detected by Canny nor the best result of $C$-ELI (see Fig. 7). With large $r$, much of the missing boundary is being detected. Overall, we believe the exponent parameters offer a powerful and flexible mechanism for detecting meaningful edges according to gradient, length or curvature, or a combination of these three, as well as achieving an global best solution according to a given evaluation criterion, such as Bayes risk or AUC.

\section{CONCLUSION}

In this paper, the problem of choosing a proper plausibility measure to distinguish low gradient edges from noise was investigated. A composite measurement composed of gradient, length, and curvature was defined, based on which an edge detection method was proposed. An extensive evaluation of the proposed method revealed that (1) it is effective in detecting meaningful edges and omitting trivial features; (2) it offers an improved Bayes risk an improved AUC when compared with the Canny detector; (3) it is insensitive to initial threshold of the input edge map as long as its reasonably low; (4) it limits delocalization error to the lowest level; (5) different edge styles may be detected by flexibility adjust the ELI exponents.

The main drawback of the proposed method is that if features due to noise were spatially connected to meaningful edges in the contour extracting stage, they will not be separated subsequently. This reduces the effectiveness of the proposed method in some images with low resolution but containing detailed features. It is our intention to deal with this problem in our future research and also investigate possible quantitative rules for the selection of exponents in the $E L I$.

\section{REFERENCE}

1. I.E. Abdou and W.K. Pratt, "Quantitative design and evaluation of enhancement/thresholding edge detectors", Proceeding of the IEEE, 67(5): 753-763, 1979.

2. M. Bertero, J. Poggio and V. Torre, "Ill-posed problems in early vision", Proceedings of the IEEE, 76(8):869-889, 1988.

3. K.W. Bowyer and P.J. Phillips, editors, Empirical Evaluation Techniques in computer vision, IEEE computer Society Press, 1998.

4. K.W. Bowyer, C. Kranenburg, and S. Dougherty, "Edge Detector Evaluation Using Empirical ROC Curves", Proc. Computer Vision and Pattern Recognition, pp 354-359, 1999.

5. J.F. Canny, "Finding edges and lines in image", Tech. Report No. 720, Massachusetts Institute of Technology, 1983.

6. J.F. Canny, "A computational approach to edge detection", IEEE Trans. Pattern Analysis and Machine Intelligence, 8(6):34-43, 1986.

7. M. Heath et al, "A robust visual method for assessing the relative performance of edge detection algorithms", IEEE Trans. PAMI 19(12):1338-1359, 1997.

8. R.M. Haralick and L.G. Shapiro, Computer and Robot Vision, Volume I, Addison-Wesley, 1992.

9. X.Y. Jiang et al, "A methodology for evaluating edge detection techniques for range images", 1995 Asian Conference on Computer Vision, vol.II: 415-419.

10. L. Kitchen and A. Rosenfeld, "Edge evaluation using local edge coherence", IEEE Trans. SMC, 11(9):597-605, 1981.

11. S. Konishi PhD Thesis, Dept. of Biophysics, Univ. of California at Berkeley, 2002.

12. S. Konishi et al, "Statistical edge detection: learning and evaluating edge cues", IEEE Trans. PAMI, 25(1):57-74, Jan 
2003.

13. P.D. Kovesi, "A Dimensionless Measure of Edge Significance", The Australian Pattern Recognition Society, Conference on Digital Image Computing: Techniques and Applications, Melbourne, 281-288, 1991.

14. P.D. Kovesi, "Image Features From Phase Congruency", Videre: A Journal of Computer Vision Research. MIT Press. 1(3), 1999.

15. D. Marr and E. Hildreth, "Theory of edge detection", Proc. R. Soc. Lond. A, Math. Phys. Sci., B207:187-217, 1980.

16. F. Mokhtarian and A.K. Mackworth, "A Theory of Multi-scale, Curvature-based Shape Representation for Planar Curves", IEEE Trans. PAMI, 14(8):789-805,1992

17. M.C. Morrone, J.R. Ross, D.C. Burr, and R.A. Owens, "Mach bands are phase dependent", Nature, 324(6094): 250-253, 1986.

18. M.C. Morrone and R.A. Owens, "Feature detection from local energy", Pattern Recognition Letters, 6:303-313, 1987.

19. P.L. Palmer, H. Dabis and J. Kittler, "A Performance measure for boundary detection algorithms", CVIU 63(3):476-494, 1996.

20. T. Poggio and V. Torre, “A regularized solution to edge detection”, Journal of Complexity, 4(2): 106-123, 1988.

21. W.K. Pratt, Digital Image Processing, New York: Wiley-Interscience, 1978.

22. A. Rosenfeld and AC. Kak, Digital Picture Processing, $2^{\text {nd }}$ ed. New York: Acadaemic, 1982.

23. M. Salotti et al, "Evaluation of edge detectors: Critics and proposal", Workshop on Performance Characterization of Vision Algorithms, 1996

24. J. Shen and S. Castan, "Toward the unification of band-limited derivative operators for edge detection", Signal Process., 31:103-119, 1993.

25. M.C. Shin et al, "An objective comparison methodology of edge detection algorithms using a structure from motion task", CVPR'98 190-195.

26. M.C. Shin, D. Goldof, and K.W. Bowyer, "Comparison of Edge Detectors Using an Object Recognition Task", Proc. Computer Vision and Pattern Recognition Conf., 360-365, 1999.

27. S. Smith and J. Brady, "A new approach to low-level image processing". International Journal of Computer Vision, 23(1):45-48, 1997.

28. D. Ziou, and S. Tabbone, "Edge detection techniques - an overview", Tech. Report No. 195, Dept. of Math et Informatique. Universit de Sherbrooke, 1997. 